\title{
Cold agglutinin disease presenting as livedo racemosa
}

\author{
Chihiro Shiiya MD, Mitsuhito Ota MD PhD
}

Cite as: CMAJ 2017 June 5;189:E781. doi: 10.1503/cmaj.161407

A n 82-year-old woman presented with a three-month history of a net-like pattern of purple patches over her entire body (Figure 1). Laboratory investigation showed signs of hemolytic anemia (reticulocyte count of $6.5 \%$ of red blood cells [normal 0.6-2.4], indirect bilirubin of $27.5 \mu \mathrm{mol} / \mathrm{L}$ (1.7-13.7), lactate dehydrogenase of $245 \mathrm{U} / \mathrm{L}$ (119-240] and undetectable haptoglobin) with a hemoglobin level of $99 \mathrm{~g} / \mathrm{L}$ (113-152). Testing for antinuclear and antiphospholipid antibodies was negative. Cryoglobulins and cryofibrinogen were not detected. Direct and indirect Coombs tests were positive, and cold agglutinins were positive at a titre of $1: 2048(<256)$ at $4^{\circ} \mathrm{C}$. Skin biopsy showed thromboses in the dermal small vessels (Appendix 1, available at www.cmaj.ca/lookup/ suppl/doi:10.1503/cmaj.161407/-/DC1). We diagnosed livedo racemosa resulting from cold agglutinin disease. There was no evidence of Mycoplasma pneumoniae infection. Immunoelectrophoresis showed immunoglobulin M (IgM)- $\lambda$ monoclonal gammopathy, but bone marrow examination did not confirm Waldenström macroglobulinemia.

While the patient was in the warm environment of the hospital, her symptoms went into remission. When she was discharged, we gave her instructions to protect herself against cold, and a hematologist is monitoring her for IgM gammopathy.

Livedo is a cutaneous cyanotic sign characterized by a net-like discoloration, and has two different forms. Livedo reticularis, which usually appears on the legs, is a primary or physiological disorder such as cold-induced vasoconstriction. Livedo racemosa, which appears in larger patches over the entire body, is a secondary vascular disorder. The differential diagnosis of the underlying disease includes antiphospholipid syndrome, systemic lupus erythematosus, cryoglobulinemia, vasculitis, thrombocythemia and cold agglutinin disease. ${ }^{1}$

Cold agglutinin disease is a hemolytic disorder induced by cold agglutinins, which are either monoclonal or polyclonal IgM autoantibodies against antigens on the erythrocyte membrane. Usually, monoclonal antibodies in cold agglutinin disease are idiopathic or secondary to lymphoproliferative disorders. Polyclonal antibodies appear in cold agglutinin disease after infections, especially those of Mycoplasma pneumoniae. The autoantibodies agglutinate erythrocytes below certain temperatures. Therefore, in addition to treating the associated underlying disorder, the primary treatment for cold agglutinin disease is maintaining the patient in a warm environment. Corticosteroids, cyclophosphamide, interferon- $\alpha$ or rituximab are occasionally helpful. ${ }^{2}$

\section{References}

1. Sajjan VV, Lunge S, Swamy MB, et al. Livedo reticularis: a review of the literature. Indian Dermatol Online J 2015;6:315-21.

2. Berentsen S, Tjønnfjord GE. Diagnosis and treatment of cold agglutinin mediated autoimmune hemolytic anemia. Blood Rev 2012;26:107-15.

\section{Competing interests: None declared.}

This article has been peer reviewed.

The authors have obtained patient consent.

Affiliation: Department of Dermatology, Chitose City Hospital, Chitose, Japan

Correspondence to: Mitsuhito Ota, ota@med.hokudai.ac.jp 